\title{
Current Patterns and Orbital Magnetism in Mesoscopic de Transport
}

\author{
Michael Walz, ${ }^{1,2,3, *}$ Jan Wilhelm, ${ }^{1,2}$ and Ferdinand Evers ${ }^{1,2,3}$ \\ ${ }^{1}$ Institute of Nanotechnology, Karlsruhe Institute of Technology, Campus North, D-76344 Eggenstein-Leopoldshafen, Germany \\ ${ }^{2}$ Institut für Theorie der Kondensierten Materie, Karlsruhe Institute of Technology, Campus South, D-76128 Karlsruhe, Germany \\ ${ }^{3}$ Center of Functional Nanostructures, Karlsruhe Institute of Technology, Campus South, D-76131 Karlsruhe, Germany
}

(Received 6 June 2014; published 26 September 2014)

\begin{abstract}
We present $a b$ initio calculations of the local current density $\mathbf{j}(\mathbf{r})$ as it arises in dc-transport measurements. We discover pronounced patterns in the local current density, ring currents ("eddies"), that go along with orbital magnetism. Importantly, the magnitude of the ring currents can exceed the (average) transport current by orders of magnitude. We find associated magnetic fields that exhibit drastic fluctuations with field gradients reaching $1 \mathrm{~T} \mathrm{~nm}^{-1} \mathrm{~V}^{-1}$. The relevance of our observations for spin relaxation in systems with very weak spin-orbit interaction, such as organic semiconductors, is discussed. In such systems, spin relaxation induced by bias driven orbital magnetism competes with relaxation induced by the hyperfine interaction and appears to be of similar strength. We propose a NMR-type experiment in the presence of dc-current flow to observe the spatial fluctuations of the induced magnetic fields.
\end{abstract}

DOI: 10.1103/PhysRevLett.113.136602

PACS numbers: 72.25.Rb, 72.80.Vp, 73.23.-b

For the physical nanosciences, the question of how current flows through a device is a vital one. The reason is obvious: sending a stream of charges through a device is the engineer's simplest way to "talk" to it. For instance, electrical currents serve as sensors in scanning tunneling microscopes, but also can operate single molecule switching in molecular electronics. A good part of the theoretical nanosciences deals with the question of how to reconstruct the detailed physics that governs at the nanoscale from the experimentally measured current-voltage (IV) characteristics. A traditional theoretical concept in this context is the Landauer-Büttiker theory, which describes charge transport as a sequence of scattering processes of (very weakly) interacting particles. The charming aspect of this approach is that it reduces the complicated transport problem to the calculation of the scattering asymptotics, incarnated in (usually a small number of) transmission coefficients. As is often the case, the reduction of complexity also eliminates interesting physics that in the present case relates to the internal structure of the scattering states. This inner structure reflects the nanoscopic pathways that the current takes through the device region.

Detailed mappings of the current flow through the sample have been attempted only relatively recently. In their ground breaking experiment, Topinka et al. monitored the resistance change $\delta \varrho(\mathbf{r})$ of a point contact embedded in a two-dimensional electron gas that is brought about by a STM tip when scanning the device region ( $\mathbf{r}$ is the tip position in the $x y$-plane) $[1,2]$. They observed a rich filamentary structure that inspired consecutive theoretical work [3]. In the course of this analysis it was emphasized that strictly speaking $\delta \varrho(\mathbf{r})$ reflects the sensitivity of the scattering states to local changes in the scattering potential $\delta V(\mathbf{r})$. Since such changes are nonlocal due to their origin in quantum interference, $\delta \varrho(\mathbf{r})$ does not directly image the actual current flow. Such flow images will be presented in this work.

Model.-The model system that we analyze is a graphene nanoribbon that has been functionalized with hydrogen as an adsorbate. Our choice is motivated in three ways. First, graphene is considered to be a key material for future technology applications, e.g., in optoelectronics. Second, graphene is hosting a two-dimensional electron gas at the material surface. Therefore, it is relatively easy for local probes, such as magnetic STMs, to access it. Third, hydrogen is a very common adsorbate.

We calculate the local current density $\mathbf{j}(\mathbf{r})$ (per spin) employing an $a b$ initio formalism based on the density functional theory (DFT) and the nonequilibrium Green's function method as implemented in our toolbox AITRANSS [4-7] which is a part of the ab initio package FHI-AIMS [8]. Using a Landauer-Büttiker approach $[9,10]$, we partition the graphene nanoribbon into a central region, a left and a right lead. We extract the single particle (Kohn-Sham) Green's function $\mathbf{G}_{0}(E)$ for the uncoupled device region from the DFT calculations. Then, we calculate the selfenergies $\boldsymbol{\Sigma}_{L / R}$ using absorbing boundary conditions and a recursive Green's function technique to model the infinite extension of our system in current direction [11,12]. The resulting Green's function

$$
\mathbf{G}(E)^{-1}=\mathbf{G}_{0}(E)^{-1}-\boldsymbol{\Sigma}_{R}(E)-\boldsymbol{\Sigma}_{L}(E)
$$

can be used to calculate the transmission through the system $\mathcal{T}(E)=\operatorname{Tr}\left\{\boldsymbol{\Gamma}_{L} \mathbf{G} \boldsymbol{\Gamma}_{R} \mathbf{G}^{\dagger}\right\}$, where the matrices $\boldsymbol{\Gamma}_{L / R}$ denote the anti-Hermitian parts of the self-energies, i.e., $\boldsymbol{\Gamma}_{L / R}=i\left(\boldsymbol{\Sigma}_{L / R}-\boldsymbol{\Sigma}_{L / R}^{\dagger}\right)$. They account for the level broadenings of the central system due to the coupling to the leads. 
Important for this work is that the Green's function allows us to calculate the Keldysh Green's function $\mathbf{G}^{<}$,

$$
\mathbf{G}^{<}=i \mathbf{G}\left[f_{L} \boldsymbol{\Gamma}_{L}+f_{R} \boldsymbol{\Gamma}_{R}\right] \mathbf{G}^{\dagger} .
$$

The Keldysh Green's function depends on the occupation numbers $f_{L / R}(E)$ of the leads. In the case of a dc-transport setup, a bias voltage $V_{\text {bias }}$ is applied between the leads. Inside the voltage window $\left(f_{L}=1, f_{R}=0\right)$, the Keldysh Green's function reduces to

$$
\mathbf{G}^{<}(E)=i \mathbf{G}(E) \boldsymbol{\Gamma}_{L}(E) \mathbf{G}^{\dagger}(E) .
$$

To calculate local observables, we transform the Keldysh Green's function to continuous real space using the basis functions $\varphi_{i}(\mathbf{r})$ of the underlying DFT calculation, $G^{<}\left(\mathbf{r}, \mathbf{r}^{\prime}, E\right)=\sum_{i j} \varphi_{i}(\mathbf{r}) \mathbf{G}^{<}{ }_{i j}(E) \varphi_{j}^{*}\left(\mathbf{r}^{\prime}\right)$. Using this decomposition, the current density (per spin) is

$$
\left.\frac{d \mathbf{j}(\mathbf{r})}{d V_{\text {bias }}}\right|_{E}=\frac{1}{2 \pi} \frac{\hbar^{2}}{2 m} \lim _{\mathbf{r}^{\prime} \rightarrow \mathbf{r}}\left(\nabla_{\mathbf{r}^{\prime}}-\nabla_{\mathbf{r}}\right) G^{<}\left(\mathbf{r}, \mathbf{r}^{\prime}, E\right) .
$$

The magnetic field $\mathbf{B}$ and the magnetization $\mathbf{m}$ induced by the current density are given by the Biot-Savart law,

$$
\begin{aligned}
\mathbf{B}(\mathbf{r}) & =\frac{\mu_{0}}{4 \pi} \int \frac{\mathbf{j}\left(\mathbf{r}^{\prime}\right) \times\left(\mathbf{r}-\mathbf{r}^{\prime}\right)}{\left|\mathbf{r}-\mathbf{r}^{\prime}\right|^{3}} d^{3} \mathbf{r}^{\prime}, \\
\mathbf{m} & =\frac{1}{2} \int \mathbf{r} \times \mathbf{j} d V .
\end{aligned}
$$

The presented Keldysh formalism is well established to describe nonequilibrium phenomena. The numerical challenges of the presented work were a high numerical cost of $a b$ initio simulations of large systems, especially because one has to ensure convergence with respect to numerical parameters [13].

Results. - An image of the current flowing in a wide ribbon is depicted in Fig. 1. It immediately confirms a suspicion that could have been based on earlier experimental work [1]: the current flow follows indeed a complicated filamentary pattern. More importantly, in Fig. 1 we discover a significant new feature: the flow has a pronounced tendency to form ring structures (eddies) with a local current strength that exceeds the (average) through current by orders of magnitude.

The strong fluctuations in the current density induce a highly inhomogeneous magnetic field with very large correlation length, see Fig. 2. Magnetic islands strongly vary in size, ranging from subatomic distances up to several nanometers.

Eddies, as seen in Fig. 1, are a signature of the mesoscopic fluctuations of wave functions in disordered media [15]. The latter manifest as reproducible fluctuations of the conductance with an amplitude of the order of one conductance quantum when control parameters like the Fermi energy or magnetic flux are varied. Such conductance fluctuations are also present in the sample seen in Fig. 1, as can be seen from the corresponding transmission curve $\mathcal{T}(E)$ shown in Fig. 3. The transmission peaks reflect

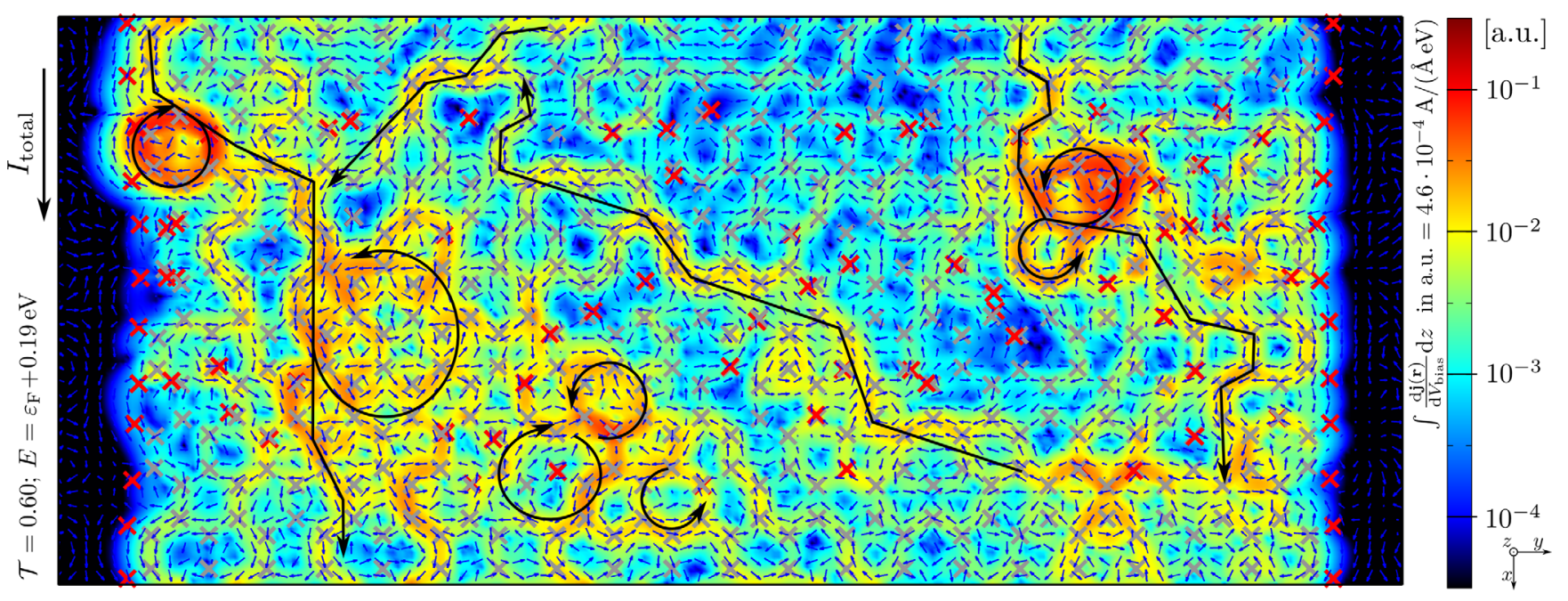

FIG. 1 (color online). Local current density per spin (integrated over the out-of-plane direction) in a wide hydrogen-terminated armchair graphene nanoribbon $(41 \times 8)$ that has been functionalized with an additional $20 \%$ hydrogen atoms (additional 66 hydrogen atoms). The current exhibits very strong mesoscopic fluctuations that reflect in a logarithmic color scale covering 4 decades. Some interesting current paths are drawn in the picture for illustration: local current vortices exceeding the spatial average current by orders of magnitude (see dark red regions; the average current is only $\left.\int\left(d j_{x}(\mathbf{r}) / d V_{\text {bias }}\right) d z\right|_{\text {avg }}=\mathcal{T}\left(e^{2} / h\right) w^{-1}=10^{-3}$ a.u.; width $\left.w=5.2 \mathrm{~nm}\right)$ and a local backflow channel where the current runs against the average current (see central arrow from bottom to top; average current direction: from top to bottom). Plot shows current amplitude (color), current direction (arrows), carbon atoms (gray crosses), and hydrogen atoms (red crosses). Sample magnetization per bias: $d m_{z} / d V_{\text {bias }}=-38$ a.u. $=-2.8\left(\mu_{B} / \mathrm{V}\right)$. (See Sec. S5 of the Supplemental Material [14] for the detailed atomic structure.) 


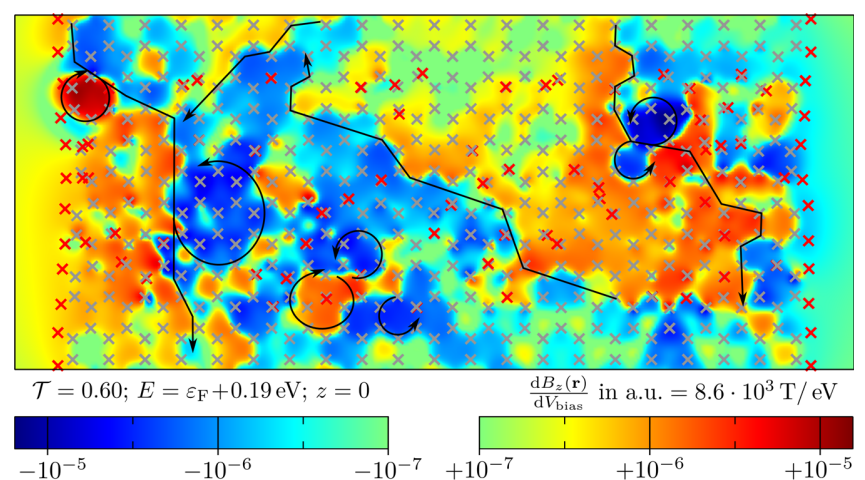

FIG. 2 (color online). Magnetic field distribution per spin (in out-of-plane direction) for the ribbon of Fig. 1. The magnetic field strongly varies and changes sign from region to region. The field is plotted in the (averaged) carbon plane $(z=0)$, but being divergence free, it hardly changes with $z$ (checked for $z= \pm 1 \AA$ ). The current paths drawn in Fig. 1 are repeated for convenience.

individual current carrying sample states. Interestingly, many maxima are seen to be separated by very sharp antiresonances.

Discussion.-A first qualitative understanding of the origin of the phenomena seen in Fig. 1 can be obtained observing that eddies and antiresonances exist already in samples with a single scatterer; they are not restricted to hydrogen adatoms. We also observe eddies and antiresonances when using $\mathrm{N}, \mathrm{B}$, and $\mathrm{OH}$ as impurities. For illustration, Fig. 4 displays a pronounced current loop that appears in a narrow armchair ribbon with a single nitrogen substituent, only. The associated transmission curve, shown in Fig. 3 (right), exhibits the antiresonance feature, i.e. a special energy at which the transmission nearly vanishes, $\mathcal{T}\left(E_{\text {res }}\right)=0$. The magnetization $d m_{z} / d V_{\text {bias }}$, corresponding to the average rotation sense of the current vortices, also vanishes at $E_{\text {res }}$ but additionally changes sign.
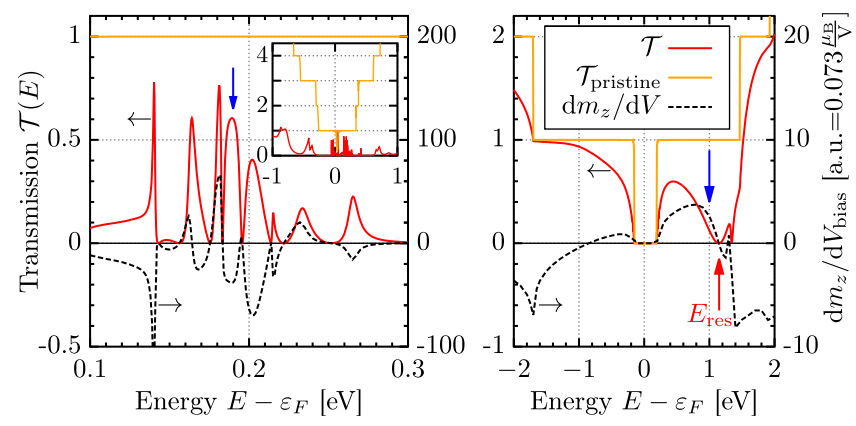

FIG. 3 (color online). The transmission functions (red, left $y$ axis) belong to the ribbons shown in Fig. 1 (left) and Fig. 4 (right). The transmission functions of the pristine ribbons show sharp steps (orange, left $y$ axis). The blue arrows mark the energy for which the current response is plotted in Figs. 1 and 5. Both ribbons exhibit a finite band gap at $E=\varepsilon_{F}$ with $\mathcal{T}=0$. The sample magnetization per bias $d m_{z} / d V_{\text {bias }}$ (perpendicular to the graphene plane, dashed black, right $y$ axis) shows sign changes in the vicinity of antiresonances.
A vanishing transmission in this setup should be understood as a consequence of quantum interference. The adsorbate splits off a resonant level from the conduction band continuum. In the present case the nitrogen contributes an additional electron to the $\pi$ bands. The associated quasilocalized state contributes a separate transmission channel that can interfere with the residual ones. In certain energy windows interference is destructive and the current is blocked.

In order to support our qualitative argument and connect back to the current loops, we consult a two-path (toy) model. The model features two current paths (upper and lower path) that connect to the same reservoir; see the Fig. 5 inset. Results of a simple analytic calculation (given in Sec. S2 of the Supplemental Material [14]) are displayed in Fig. 5. It is comforting to see that a well known fact is reproduced [16]: antiresonances (destructive interference) are a generic encounter in this model. The transmission $\mathcal{T}$ vanishes at the antiresonance $E_{\text {res }}=\left(\varepsilon_{T}+\varepsilon_{B}\right) / 2$.

The new aspect relevant to us is that the response of the loop current (orbital magnetization) near $E_{\text {res }}$ is linear in the detuning $E-E_{\text {res }}$ while the through current (transmission) is quadratic, see Fig. 5; compare also Fig. 3 (right). Hence, the ratio of orbital current (magnetization) to the transport current becomes arbitrarily large near the antiresonance $E_{\text {res }}$. Moreover, at the resonance the loop current changes its direction emphasizing the close relation of the effect to quantum interference.

Observable consequences.-After discussing the microscopic origin of the loop currents, we turn our attention to
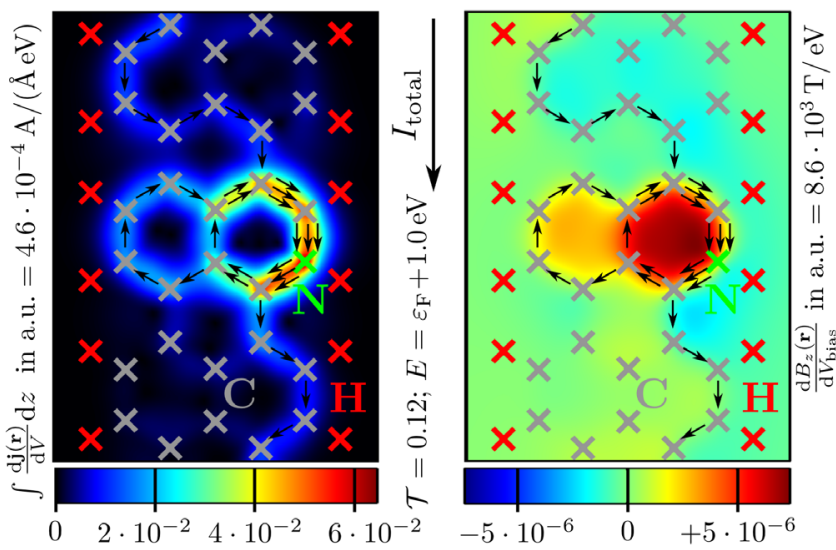

FIG. 4 (color online). Local current density per spin (left) and induced magnetic field (right) in a narrow armchair ribbon (five carbon atoms wide) with one nitrogen atom replacing a carbon atom (at right edge) The current reveals a massive tendency for (local) vortices that exceeds the spatial average of the current density (through current) by an order of magnitude. The average current is only $\int\left(d j_{x}(\mathbf{r}) /\right.$ $\left.d V_{\text {bias }}\right)\left.d z\right|_{\text {avg }}=\mathcal{T}\left(e^{2} / h\right) w^{-1}=1.5 \times 10^{-3}$ a.u.; $\quad$ width $\quad w=$ $6.8 \AA$ A). Average current direction: from top to bottom. Sample magnetization per bias: $d m_{z} / d V_{\text {bias }}=2.6$ a.u. $=$ $0.19 \mu_{B} / \mathrm{V}$. 


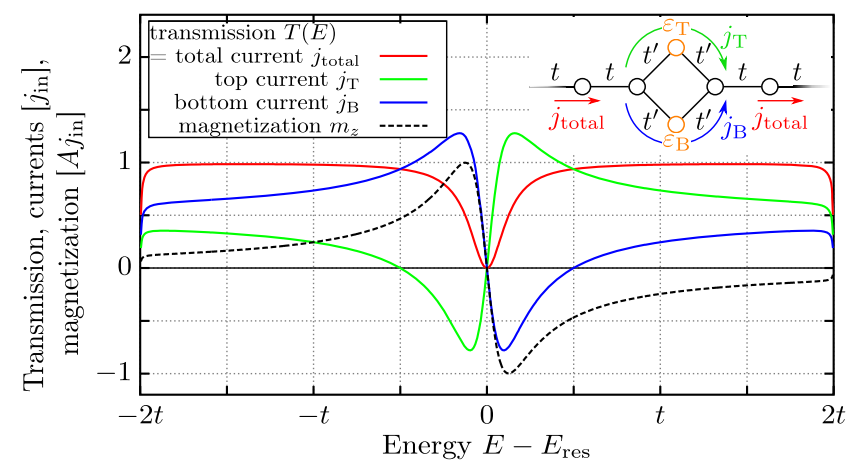

FIG. 5 (color online). Transport current and orbital current (magnetization) as calculated for the two-path (toy) model. Currents are proportional to the incoming current $j_{\text {in }}$. Inset: graphical representation of the (tight-binding) two-path model. $\left(t^{\prime}=t / \sqrt{2} ; \varepsilon_{T}=-\varepsilon_{B}=\frac{1}{2} t ; m_{z}=\left(j_{B}-j_{T}\right) A / 2\right)$.

observable consequences. First, we recall that random magnetic fields give rise to spin relaxation. In leading order perturbation theory the corresponding rate reads $\tau_{s}^{-1} \sim\left(\mu_{B} B_{0}\right)^{2} \tau$, where $B_{0}$ is the typical strength of the random magnetic field and $\tau$ denotes the time it takes the electron to move from one current loop to the next [17]. For the example depicted in Fig. 2, we could expect a bare value for $\mu_{B} B_{0} \sim 100 \mathrm{MHz}$ at $V_{\text {bias }}=10 \mathrm{meV}$. This value is in the range typical of hyperfine interactions. Such nominally weak interactions might become the relevant source of spin relaxation in organic materials where the competing relaxation mechanism, spin-orbit interaction, may be quite weak. In particular, for strongly disordered films, the relaxation rate $\tau_{s}$ formally diverges due to correlated multiple scattering processes (i.e., current loops) [18]. (Notice, however, that recent works indicate that spin-orbit interaction may be significant in hydrogenated graphene, enhanced by lattice distortion [19].)

Second, we follow the analogy with the hyperfine structure and ask what follows from the existence of bias driven loop currents for NMR experiments performed on molecular nanostructures in the presence of current flow. In addition to the chemical shift due to the chemical environment, the observed NMR shifts depend on the induced local magnetic field. If we think about the functional part of the current carrying device as a big molecule, then we might hope to reconstruct from conventional NMR technology (or otherwise) its atomistic structure. If this is possible, then one also can follow the shift of the NMR resonances that results from the loop currents upon slowly increasing the bias voltage. Since this shift directly measures the (bias induced) local magnetic field, one can in principle measure the orbital current flow with atomic precision in a global experiment.

Third, we would like to emphasize that we expect loop currents to exist in generic situations with phase-coherent charge transport in the presence of broken symmetries, such as those brought about by disorder. As illustrated by tight-binding (toy) models, the formation of current vortices is forbidden in the presence of inversion or mirror symmetries (see Secs. S3 and S4 of the Supplemental Material [14]). Experimentally, such perfect symmetries are typically broken and current vortices should be generic encounters.

Outlook.-We conclude with an outlook. The idea underlying this work is very general: scattering states of mesoscopic samples have an inner, nontrivial structure. Our focus here was on implications for orbital magnetism, but the theme readily generalizes to other observables as well. As an example we mention heat: Meair et al. have recently proposed the concept of a local temperature applicable to an out-of-equilibrium situation [20]. This beautiful new concept is understood from the point of view advocated in this Letter in the following way. A mesoscopic device, such as a graphene nanoribbon, has contributions to the local particle density made by scattering states that emanate from right and left reservoirs. If the reservoirs carry different temperatures, the sample will exhibit local temperature fluctuations. Hot regions have predominant charge contributions from the hot electrode, while colder regions receive the majority of carriers from the other electrode.

We express our gratitude to Alexei Bagrets for helpful discussions. We acknowledge the DFG (EV30/7-1 and EV30/8-1) for financial support. We also express our gratitude to the Simulation Lab NanoMicro, especially to I. Kondov, for computational support. The authors gratefully acknowledge the computing time granted by the High Performance Computing Center Stuttgart (HLRS) on the HERMIT supercomputer, and the computing time on the HC3 cluster at the Karlsruhe Institute of Technology (KIT) operated by the Steinbuch Center for Computing (SCC).

*michael.walz@kit.edu

[1] M. A. Topinka, B. J. LeRoy, S. E. J. Shaw, E. J. Heller, R. M. Westervelt, K. D. Maranowski, and A. C. Gossard, Science 289, 2323 (2000).

[2] M. A. Topinka, B. J. LeRoy, R. M. Westervelt, S. E. J. Shaw, R. Fleischmann, E. J. Heller, K. D. Maranowski, and A. C. Gossard, Nature (London) 410, 183 (2001).

[3] R. A. Jalabert, W. Szewc, S. Tomsovic, and D. Weinmann, Phys. Rev. Lett. 105, 166802 (2010).

[4] A. Bagrets, J. Chem. Theory Comput. 9, 2801 (2013).

[5] A. Arnold, F. Weigend, and F. Evers, J. Chem. Phys. 126, 174101 (2007).

[6] J. Wilhelm, M. Walz, M. Stendel, A. Bagrets, and F. Evers, Phys. Chem. Chem. Phys. 15, 6684 (2013).

[7] J. Wilhelm, M. Walz, and F. Evers, Phys. Rev. B 89, 195406 (2014).

[8] We performed spin-unpolarized DFT simulations using FHI-AIMS [21] applying a generalized gradient approximation (GGA) using numerical tabulated tier 1 basis functions (14 basis functions per carbon atom, 5 per hydrogen, Perdew-Burke-Ernzerhof [PBE] exchange-correlation 
functional). We checked that a spin-polarized DFT simulation with an initial moment of $79.4 \mu_{B}\left(1 \mu_{B}\right)$ converges to the spin-unpolarized solution [for the ribbon of Fig. 1 (Fig. 4)]. The graphene ribbons in this Letter were structurally relaxed until the force decreased below $10^{-2}(\mathrm{eV} / \AA)$. The self-energies were calculated using 200 recursion steps. The current of Eq. (4) was calculated in a three-dimensional spatial grid with a grid spacing of 0.2 and $0.1 \AA$ for the ribbon of Figs. 1 and 4, respectively. These grids were reused to numerically calculate the integrals of Eqs. (5). For convergence studies regarding our transport method please refer to Ref. [12].

[9] R. Landauer, IBM J. Res. Dev. 1, 223 (1957).

[10] M. Büttiker, Y. Imry, R. Landauer, and S. Pinhas, Phys. Rev. B 31, 6207 (1985).

[11] F. Evers and A. Arnold, in Nanoelectronics, edited by C. Röthig, G. Schön, and M. Vojta, CFN Lectures on Functional Nanostructures Vol. 2 (Springer, Berlin, 2011).

[12] M. Walz, A. Bagrets, and F. Evers (unpublished).

[13] One important numerical parameter to check is the basis set size since the transformation of the Green's function to continuous real space is only exact in the limit of an infinite basis set. The number of basis functions used for the ribbon in Fig. 1 is 9874. The necessary convergence check calculation with a larger (tier 2) basis set already contained 27654 basis functions. To deal with such large basis set sizes, the DFT simulation and the transport calculation need to be massively parallelized, see Refs. [12,21-23] for details. The structural relaxation of the ribbon in Fig. 1 took 12000 core hours (using 256 cores on the HC3 cluster) and the calculation of all transmission values, local currents, magnetic fields plotted in or needed for Figs. 1, 2, and 3 took 43000 core hours (using 768-2048 cores on the HERMIT cluster).

[14] See Supplemental Material at http://link.aps.org/ supplemental/10.1103/PhysRevLett.113.136602 for the analytic solution of the two-path (toy) model shown in the inset of Fig. 5, an overview of additional tight-binding (toy) models supporting or prohibiting ring currents, and additional information about the atomic structure and the self-energies of the graphene nanoribbon shown in Figs. 1 and 2.

[15] K. Efetov, Supersymmetry in Disorder and Chaos, 1st ed. (Cambridge University Press, Cambridge, England, 1999).

[16] G. Géranton, C. Seiler, A. Bagrets, L. Venkataraman, and F. Evers, J. Chem. Phys. 139, 234701 (2013).

[17] M. I. Dyakonov and V. I. Perel, Sov. Phys. Solid State 13, 3023 (1971).

[18] R. C. Roundy and M. E. Raikh, arXiv:1401.4796.

[19] M. Gmitra, D. Kochan, and J. Fabian, Phys. Rev. Lett. 110, 246602 (2013).

[20] J. Meair, J. P. Bergfield, C. A. Stafford, and P. Jacquod, Phys. Rev. B 90, 035407 (2014).

[21] V. Blum, R. Gehrke, F. Hanke, P. Havu, V. Havu, X. Ren, K. Reuter, and M. Scheffler, Comput. Phys. Commun. 180, 2175 (2009).

[22] V. Havu, V. Blum, P. Havu, and M. Scheffler, J. Comput. Phys. 228, 8367 (2009).

[23] A. Marek, V. Blum, R. Johanni, V. Havu, B. Lang, T. Auckenthaler, A. Heinecke, H.-J. Bungartz, and H. Lederer, J. Phys. Condens. Matter 26, 213201 (2014). 